O brother, where art thou located? : Raising awareness of variability in location tracking for users of location-based pervasive applications Peer-reviewed author version

AKSENOV, Petr; LUYTEN, Kris \& CONINX, Karin (2012) O brother, where art thou located? : Raising awareness of variability in location tracking for users of location-based pervasive applications. In: Journal of Location Based Services, 6(4), p. 211-233..

DOI: $10.1080 / 17489725.2012 .682098$

Handle: http://hdl.handle.net/1942/13892 


\section{O brother, where art thou located?: Raising awareness of variability in location tracking for users of location-based pervasive applications}

Petr Aksenov, Kris Luyten, Karin Coninx

Hasselt University, Belgium

\{petr.aksenov, kris.luyten, karin.coninx \}@uhasselt.be

Postal address: Expertise centre for Digital Media, Wetenschapspark 2, 3590

Diepenbeek, Belgium

Tel: +32 112684 11; Fax: +3211268499

Corresponding author: kris.luyten@uhasselt.be

This is a preprint of an article whose final and definitive form has been published in the Journal of Location Based Services (C) 2012 Taylor \& Francis; Journal of Location Based Services is available online at:

http://www.tandfonline.com/doi/abs/10.1080/17489725.2012.682098 


\title{
$O$ brother, where art thou located?: Raising awareness of variability in location tracking for users of location-based pervasive applications
}

\author{
There is a diversity of ways to determine a user's location in a pervasive \\ environment today. On a large-scale, this diversity often results in variability of \\ location tracking conditions throughout the environment. For an important class \\ of pervasive applications, which often rely on the ubiquitous availability of \\ location tracking - location-based pervasive applications, the consistency of their \\ behaviour under this variability cannot be guaranteed. This type of limitation \\ raises a need for the adaptation of the application's behaviour that would reflect \\ this variability. We investigated empirically how visualising different aspects of \\ uncertainty about location and of the behaviour of localisation systems affects \\ users' impressions about a location-based application. The two components - an \\ ontology that models properties of localisation systems and a set of mapping \\ rules that define how these properties should be visualised in a user interface - \\ are at the core of our approach to providing awareness. The results of the \\ investigation show that the additional visual demand, intended for raising users' \\ awareness of uncertainty about their location tracking conditions, is perceived to \\ be beneficial by users. We reveal also that different characteristics of this \\ awareness are of different importance to users. Furthermore, we conclude that the \\ particular importance depends on users' personal profiles (e.g., their eyesight \\ level), on the distance between the users (e.g., knowing about someone else's \\ state is less important if they are far), and on the quality of tracking (the \\ importance increases in problematic areas). Based on the obtained results and \\ observations, we suggest a set of guidelines and visualisations which could be \\ used in designing pervasive applications that require location tracking.
}

Keywords: localisation systems; pervasive computing; uncertainty-awareness; visualisation

\section{Introduction}

Location is often considered the most important type of context (Varshavsky and Patel 2009). The increased popularity of location-based services and location-aware

This is a preprint of an article whose final and definitive form has been published in the Journal of Location Based Services (C) 2012 Taylor \& Francis; Journal of Location Based Services is available online at: http://www.tandfonline.com/doi/abs/10.1080/17489725.2012.682098 
applications has led to the demand that the information on users' location be available ubiquitously: everywhere and at any time. Although there are widely used solutions to location determination, such as GPS, Skyhook (http://www.skyhookwireless.com), or Ekahau (http://www.ekahau.com), providing ubiquitous and seamless location support that users expect today is a cumbersome task, mainly influenced by incompatibilities and limitations of different systems. Attempts are still being made at enhancing or combining existing technologies in order to make them cover wider areas (Hansen et al. 2009, Kjærgaard et al. 2010) or at providing peer-to-peer localisation (Banerjee et al. 2010, Gellersen et al. 2010, Wagner and Kray 2010). And the reality is that information about location still comes in different formats and via different communication channels.

Besides, every context - and thus location as well - comes with uncertainty (Dey et al. 2002, cited in Dearman et al. 2007, p. 183). Ranging from accurate and reliable in one area to insufficient, unreliable, or even completely unavailable in another, the variability of information about location influences a location-aware application's behaviour. This, in turn, affects the user experience when using such applications, so addressing uncertainty that is present in location-based experiences is important. Benford et al. (2006) suggested several strategies to cope with this uncertainty: remove it, hide it, manage it, reveal it, and exploit it. As the number of situations and mobile applications, for which the knowledge about a user's location is beneficial, has grown recently, the above research question has only gained more attention. This resulted in many approaches to handle uncertainty about location and localisation in one way or the other: based on simple logic (Damián-Reyes et al. 2011), ontology-based (Stevenson et al. 2010), probabilistic (Ranganathan et al. 2004),

This is a preprint of an article whose final and definitive form has been published in the Journal of Location Based Services (C) 2012 Taylor \& Francis; Journal of Location Based Services is available online at: http://www.tandfonline.com/doi/abs/10.1080/17489725.2012.682098 
representing it as a seam (within a positioning middleware) that the developers could exploit (Langdal et al. 2010), or adjusting the available location sensing technology according to the task at hand (Pulkkinen et al. 2011). Our previous work (Aksenov et al. 2011) also falls into the category of research initiatives on the representation and management of uncertainty about location. We introduced an ontology that models the interiors of localisation systems and used that model to build a unified view on localisation on a large-scale, with a separate focus on uncertainty and variability in location sensing and their presentation to the user.

Despite the fact that non-visual forms of providing location-awareness in a pervasive environment, such as audio and haptic feedback, have become popular (Anastassova et al. 2010), the role of visual feedback obviously remains important. Based on the unified approach mentioned above, the current work presents a mechanism for ontology-powered adaptation of the representation of a user's location in a graphical user interface. The ontology that describes the localisation systems in use is mapped onto a set of user interface elements that visualise the information about users' location sensing at run-time. We evaluated our approach in a real-life field trial on a chasing task for two mobile users, in which we explored how awareness about tracking assisted runtime navigation. The results revealed that additional visual demand reflecting users' location tracking conditions was perceived beneficial. We also found that different components of that awareness turned out to be of different importance to users. Based on the obtained results and observations, we came up with a number of design implications for location-aware graphical user interfaces that take into account the variability of location sensing.

This is a preprint of an article whose final and definitive form has been published in the Journal of Location Based Services (C) 2012 Taylor \& Francis; Journal of Location Based Services is available online at: http://www.tandfonline.com/doi/abs/10.1080/17489725.2012.682098 


\section{Related Work}

\subsection{Visualising uncertainty about location}

In some situations, uncertainty about location can be predicted and hence incorporated into the application at design-time. For example, Benford et al. (2006) used the positions of the GPS satellites at a specific moment for visualising the likely availability of GPS over a certain area at that moment; in particular, shading all buildings permanently black due to unavailability of GPS indoors at all times. But some factors about uncertainty can only become known when they appear, so that they need to be processed and visualised at run-time using the information at hand. For example, Dearman et al. (2007) explored the variability of the location error of a GSM-based localisation in outdoor map-based navigation. They represented the error as a circle projected on the map and centred at the predicted location. The radius varied depending on the level of confidence that the true position was within that circle. The results revealed that showing the error was beneficial in terms of both the perceived difficulty of completing a location finding task and the time required to do it. Lemelson et al. (2008) conducted a paper-based survey that compared several alternatives to visualising the error of users' position indoors. The respondents were asked to choose the most suitable visualisation in each of a number of scenarios. The results uncovered a clear preference for the suggested in-map visualisations (a coloured room or area on the map, or a circle-based) in each scenario. The above examples assumed that the location tracking was always available. Alternatively, Burigat and Chittaro (2011) did not make this assumption and investigated user preferences towards visualising the uncertainty caused by a degraded GPS signal. The authors compared three approaches: a basic

This is a preprint of an article whose final and definitive form has been published in the Journal of Location Based Services (C) 2012 Taylor \& Francis; Journal of Location Based Services is available online at: http://www.tandfonline.com/doi/abs/10.1080/17489725.2012.682098 
visualisation showing the predicted position only, a circle centred in the predicted position and extending proportionally to the speed of walking, and street segments, i.e. the routes along which the participants could actually walk, coloured also according to the walking speed. The participants were asked to walk along a path, where unavailability of the GPS signal was simulated in a predefined area, and pinpoint their location on the map upon request in the end. The results of the study revealed that the two uncertainty-aware visualisations were perceived to be beneficial, requiring a lower mental demand for the street-colouring and a lower effort for both.

Figure 1. In the circle-based visualisation, the predicted location is surrounded by a circular area of the positioning error.

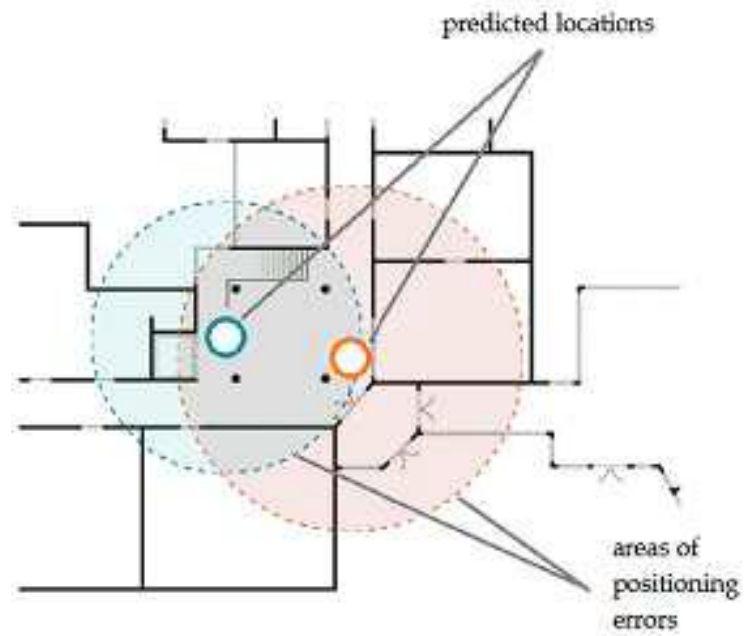

Altogether, these examples illustrate that the omnidirectional representation of positional uncertainty as a circular region, which is centred at the predicted position and with the radius corresponding to the error of a position estimate (see Figure 1), is a popular visualisation that can also be met in many other applications (e.g., Google Maps for mobile (http://www.google.com/mobile/maps/)). Others proposed other ways of

This is a preprint of an article whose final and definitive form has been published in the Journal of Location Based Services (C) 2012 Taylor \& Francis; Journal of Location Based Services is available online at: http://www.tandfonline.com/doi/abs/10.1080/17489725.2012.682098 
uncertainty visualisation that reflect the nature of the localisation system together with the actual infrastructure of the environment the user is in (e.g., Baus and Kray 2002, Burigat and Chittaro 2011, Lemelson et al. 2008). However, their comparison with the circle-based representation did not reveal preference of one over others (Burigat and Chittaro 2011, Lemelson et al. 2008), but it was preferred to alternative, i.e. not in-map, ways (Lemelson et al. 2008). Therefore we also use a circle-based visualisation to represent uncertainty. We further extend the visualisation to reflect the finer localisation dynamics in situations with several tracking providers in a mixed indoor-outdoor area, and without restrictions on navigating paths. For this purpose, we also consulted with a professional graphic designer and explained which details and situations we wanted to visualise. Section 3.1 describes the resulting extension in detail.

\subsection{Ontology-powered adaptation of pervasive applications}

Ontologies have been successfully used to represent the knowledge about one or several domains of interest while allowing developers to reason about this information. For example, Strobbe et al. (2007) presented CASP, a context-aware service platform for combining and organising context information of diverse types with the help of ontologies. They then used their platform to help determine an employee's most likely location by means of reasoning about the statuses and approximate locations of this employee's and their colleagues' personal devices (such as their laptop, PDA, or mobile phone), which were represented as an ontology. Ongenae et al. (2008), also using

CASP, demonstrated how the performance of a hospital's nurse call system improved after a place-oriented approach had evolved into a person-oriented approach. This approach additionally included reasoning about such information as urgency of a

This is a preprint of an article whose final and definitive form has been published in the Journal of Location Based Services (C) 2012 Taylor \& Francis; Journal of Location Based Services is available online at: http://www.tandfonline.com/doi/abs/10.1080/17489725.2012.682098 
patient's call or a nurse's current status. Cearreta and Garay-Vitoria (2011) aimed at adapting interfaces of ubiquitous services to users with special needs. They introduced an ontology of users' sensorial and perceptual capabilities and demonstrated how it could be used to determine and generate an appropriate means of interaction with the service automatically. Hervás and Bravo (2011) have shown that the potential of combining knowledge about several domains to provide ontology-powered proactive adaptation of pervasive user interfaces is high. By means of combining domain, device and user ontologies, the authors personalised the information shown to the requestor on a public display at run-time. The evaluation of the proposed run-time personalisation revealed that the required documents were selected in 80.77 percent of the cases.

The applications in the above examples relied on the permanent availability of location tracking. In a pervasive environment, which is usually heterogeneous and dynamic, the behaviour and availability of localisation systems change frequently. Therefore adaptation mechanisms must take this feature of the environment into account as well. In our work, we use an ontology that describes the properties and the behaviour of localisation systems and introduce user interface adaptation that reflects the changes in the localisation conditions.

\subsection{Influence of location-awareness}

We discussed in session 2.1 that revealing the error of a position estimate is beneficial. However, location awareness contains and depends on many factors, of which some may matter more than the others, some may be considered either helpful or hurtful, and some will just not make any difference.

This is a preprint of an article whose final and definitive form has been published in the Journal of Location Based Services (C) 2012 Taylor \& Francis; Journal of Location Based Services is available online at: http://www.tandfonline.com/doi/abs/10.1080/17489725.2012.682098 
For example, Dearman et al. (2005) found out that otherwise helpful awareness about rendezvousing partners' location and movements became detrimental if one of them could not determine what their partner's problem was. Similarly, Lim and Dey (2011) revealed recently that the same and previously useful awareness became harmful when the certainty of information provided in the same scenario decreased. However, the results were produced by Amazon's Mechanical Turks and thus provided passive judgements only, so these authors still plan to validate their hypotheses in a real-world scenario. Misund et al. (2009) reported that revealing information about locations of other players in a collaborative location-aware chase-and-catch game did not affect the performance in the main task; however, it did make the game more 'fun' to play. Nova et al. (2010) found out that automatic mutual location awareness made the coordination process within the tested group less efficient, as opposed to the groups whose members used self-reporting on their whereabouts.

Thus, understanding the effect and the level of importance of clues on uncertainty about location from the user point of view is useful for developers and designers of pervasive location-aware applications. We consider a set of factors about the behaviour of run-time localisation and explore how users react to changes in it. In particular, we first introduce and explain in detail (section 3) how such properties as the accuracy, the frequency, and the availability status of a user's location determination can be reflected in the graphical user interface of a map-based application at run-time. We then evaluate, in a real-life field study throughout a suggested area (section 4), how this dynamic visualisation affects navigation strategies of the users of the application while they are trying to chase a moving object.

This is a preprint of an article whose final and definitive form has been published in the Journal of Location Based Services (C) 2012 Taylor \& Francis; Journal of Location Based Services is available online at: http://www.tandfonline.com/doi/abs/10.1080/17489725.2012.682098 


\section{Presenting uncertainty about location in a user interface}

\subsection{From location tracking to visualisation patterns}

Benford et al. (2006, p. 122) discern four 'states of being' of a mobile user: connected and tracked, connected but not tracked, tracked but not connected, and neither connected nor tracked. These states separately consider the tracking of users' location (tracked) and the ability to communicate information, including location, to other mobile users (connected). Based on this classification, we introduce a set of states of awareness about the status of a mobile user's location that reflect the behaviour of an active localisation system and take into account uncertainty. They are based on an omnidirectional circular visualisation. The argumentation for this choice was explained in section 2.1. Figure 2 shows the four states that read as follows:

- (a) - a location update is received with an initial positioning error as the circle's radius, and the dot in the centre starts pulsating (see Figure 2(a));

- (b) - the radius extends as a function of the time elapsed since the last update, according to a user's moving speed. If the timeout for receiving the next update has been exceeded, the dot no longer appears (see Figure 2(b));

- (c) - if the second timeout has been exceeded, the position marker turns into a cross (see Figure 2(c));

- (d) - the error area continues to extend until the maximum age for the referred location measurement is reached. After this, the uncertainty area is no longer shown, the cross thickens, and the information about this location is considered outdated and unreliable (the visualisation becomes static) (see Figure 2(d)).

This is a preprint of an article whose final and definitive form has been published in the Journal of Location Based Services (C) 2012 Taylor \& Francis; Journal of Location Based Services is available online at: http://www.tandfonline.com/doi/abs/10.1080/17489725.2012.682098 
Thus, there are several independent elements that contribute to the visualisation in each state - a dot, a circle, and a cross. We will hereafter refer to these elements as visualisation patterns. To determine an appropriate visualisation, the tracking state is mapped onto a subset of these patterns. The decision about the appropriateness of inclusion of each pattern into the visualisation is based on this pattern's validity and importance. The validity is determined by the state of the referred location update, and the importance is either specified by the designer or determined by considering this element in connection with other context models (see section 3.3).

As part of our ontology of localisation systems that we referred to in section 1, we defined a separate concept of a single location update; hence of a user's location tracking state. Each location update has a set of common attributes that are independent of the technology used, contributing to the unified model of user localisation. In the scope of this work, the following attributes are considered: $2 D$ position, position estimate error, and the update rate, i.e. how often the location update is produced. Also, within the context of the study described in section 4, we assigned the walking speed $\mathrm{V}=1.2 \mathrm{~m} / \mathrm{s}$, which is around the lower end of the scale of the average walking speed (Patricia 2010). The timeouts for the state transitions in the case of missing updates are 5,10 , and 20 seconds, corresponding to 6,12 , and 24 metres of walking.

This is a preprint of an article whose final and definitive form has been published in the Journal of Location Based Services (C) 2012 Taylor \& Francis; Journal of Location Based Services is available online at: http://www.tandfonline.com/doi/abs/10.1080/17489725.2012.682098 
Figure 2. Visualisation of the status of a user's location tracking distinguishes four states of awareness: (a) a regular update is received; (b) an update is missing; (c) location tracking is possibly unavailable; (d) location information is outdated (visualisation is static).

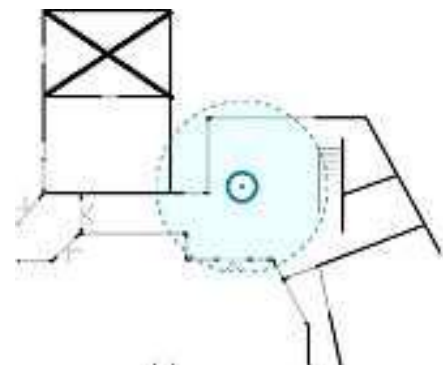

(a)

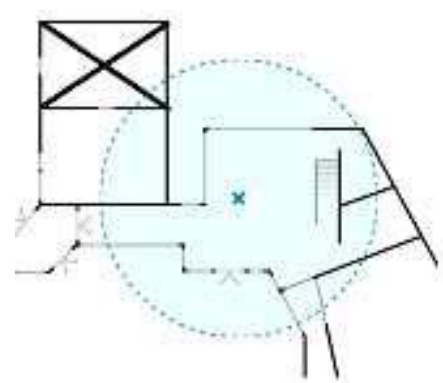

(c)

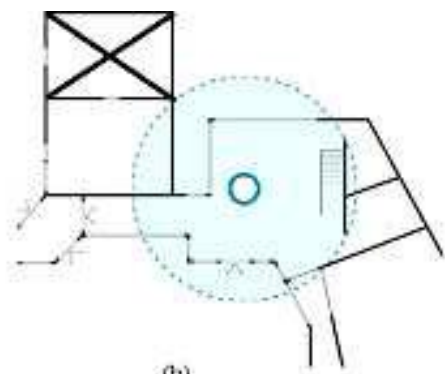

(b)

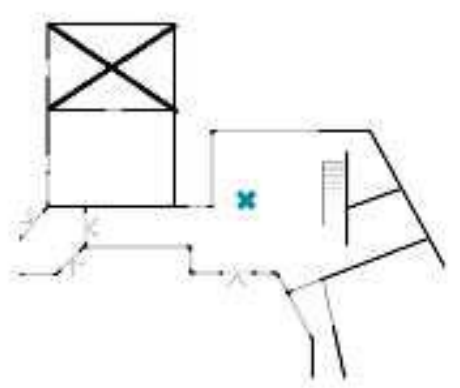

(d)

The proposed location-driven transformation of the visualisation of a user's position reflects the variability of run-time location sensing in a way that will prepare the users for a possible change in their tracking and the application's behaviour. The visual dynamics of the transition between the states informs the user about the upcoming change and, by making the transition smooth, helps to preserve the continuity of their interaction with the application. We describe in the next section how appropriate visualisations of the users' location states from Figure 2 are determined by means of applying a corresponding rule to the given location tracking conditions.

This is a preprint of an article whose final and definitive form has been published in the Journal of Location Based Services (C) 2012 Taylor \& Francis; Journal of Location Based Services is available online at: http://www.tandfonline.com/doi/abs/10.1080/17489725.2012.682098 


\subsection{System architecture}

Figure 3 depicts the flowchart of visualisation generation. Three kinds of requests to generate the visualisation are possible: two of them would query the ontology and one would be handled locally within the application.

A request of the first type is triggered when an update ( $L^{\prime}$ in Figure 3) on a user's location has been received and needs to be communicated. This update is first processed accordingly by the Location Processing Component (introduced and described in (Aksenov et al. 2011); hereafter abbreviated to LPC), which updates the ontology and then sends the updated instance to the mapping table (L to UI Mapping Table in Figure 3). The mapping table is a collection of rules that determine which subset of visualisation patterns corresponds to the attributes of the received location update. The table generates an appropriate subset (UI_SET in Figure 3) and sends it to the application that eventually adds the corresponding visualisation to the user interface.

A mapping request of the second type queries the ontology for the available details on location and state of some particular user or a group of users (e.g., in Figure 4, the user of a client application chooses to look at the map of an area with id 'main_building'). In this case, LPC processes the corresponding query and sends the results to the mapping table that does the selection.

Finally, a mapping request of the third type is activated in the case when there are no updates on the location tracking of a currently visualised user. The application sends this user's attributes ( $\mathrm{T}$ in Figure 3 ) to the mapping table that determines a degraded version of the visualisation.

This is a preprint of an article whose final and definitive form has been published in the Journal of Location Based Services (C) 2012 Taylor \& Francis; Journal of Location Based Services is available online at: http://www.tandfonline.com/doi/abs/10.1080/17489725.2012.682098 
Figure 3. A mapping to generate a visualisation is requested 1) when a location update for a currently shown user has been received (case L'), 2) a previously inactive user or the map of a different area need to be visualised (case <ID $\mid$ MAP $\mid . . .>$ ), 3) a currently shown user does not receive any updates (case T (ID)). The UI Mapping Table then matches the received parameters to a corresponding rule that determines an appropriate set of user interface visualisation patterns. Finally, the returned UI_SET is sent to the application. In the figure, the generation of the visualisation for a newly received update (case $\mathrm{L}^{\prime}, \mathrm{t}=0$ in $\mathrm{L}$ ) is illustrated, i.e. the UI_SET receives the $\{\mathrm{P}+, \mathrm{C}(\mathrm{R}+\mathrm{tV})\}$ combination of the visualisation patterns to be visualised.

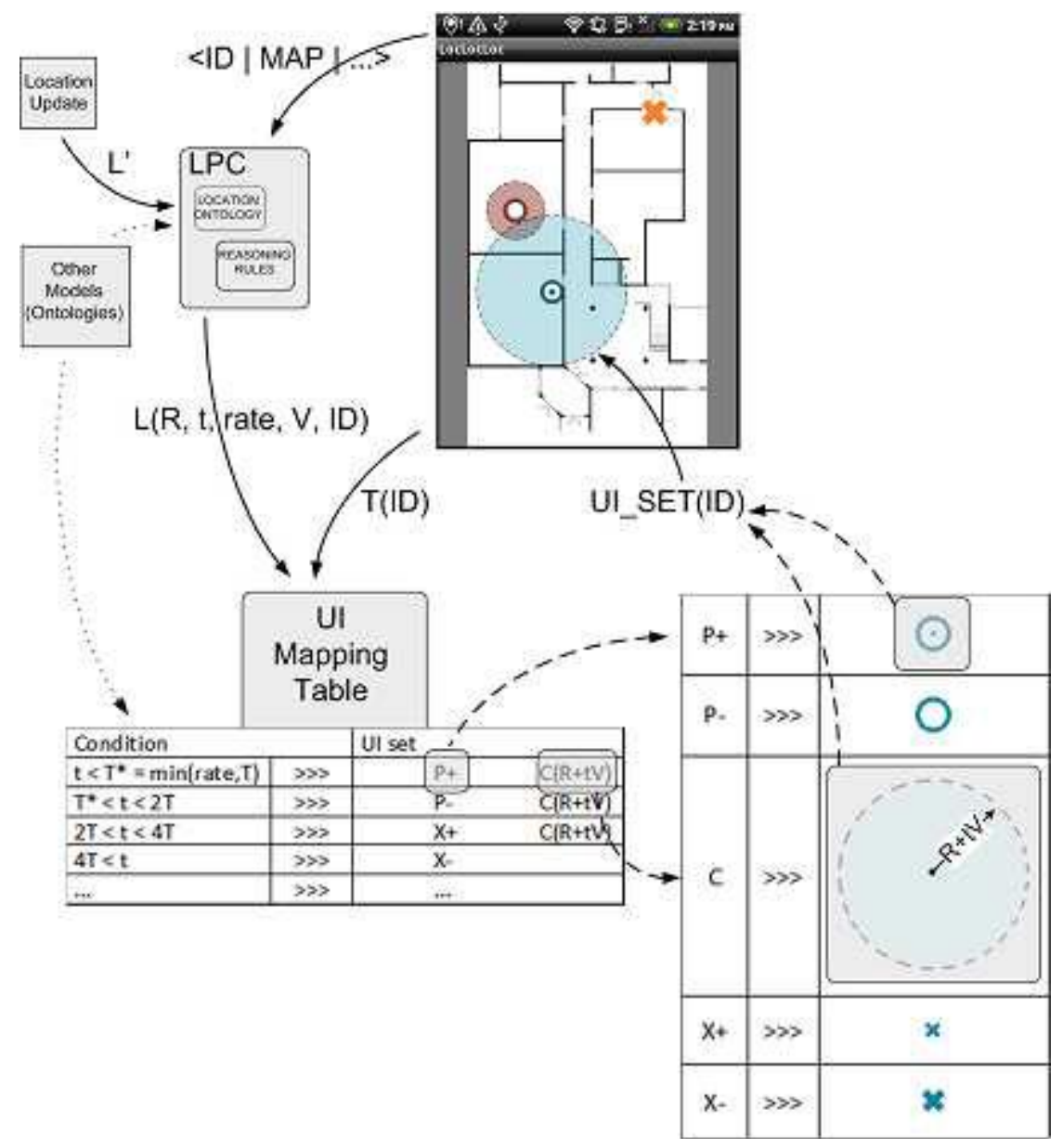

This is a preprint of an article whose final and definitive form has been published in the Journal of Location Based Services (C) 2012 Taylor \& Francis; Journal of Location Based Services is available online at: 
Figure 4. A sample SPARQL query that asks the localisation ontology for location tracking details of all users who belong to the specified map ('main_building').

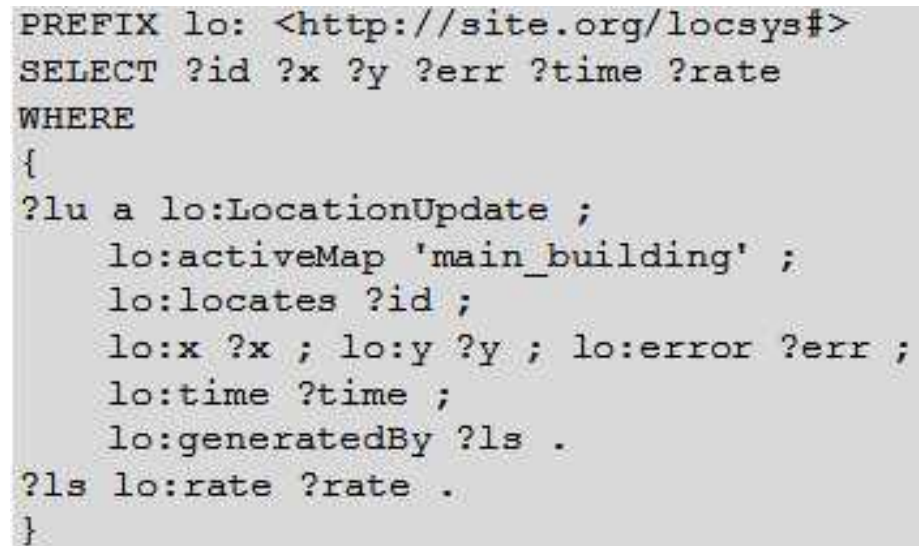

We would like to note here that all improvements and decisions on the quality and validity of the provided update are entirely in the hands of LPC. The extensions on the visual awareness introduced here do not influence the availability or the quality of localisation itself - they only reveal its details, existing problems and limitations to the user and in accordance with this user's needs.

\subsection{Other visualisation patterns and context models}

Within the scope of this work we consider a set of visualisation patterns that reflect the corresponding properties of the localisation systems involved in the field study described in section 4. Visualisation patterns exist individually and independently from one another, so other patterns that reflect other properties and states can be freely included into the mapping. For example, Tang et al. (2011) experimented with the circle's border width, colour and transparency level to distinguish between locations visited by users in the past. Marquardt et al. (2010) also described how several separate

This is a preprint of an article whose final and definitive form has been published in the Journal of Location Based Services (C) 2012 Taylor \& Francis; Journal of Location Based Services is available online at: http://www.tandfonline.com/doi/abs/10.1080/17489725.2012.682098 
elements formed the visualisation of a device in their Visual Environment Explorer application. They then discussed and illustrated how each element behaved and changed so as to reflect the availability and recent activity of the device over different time intervals and levels of detail.

Besides, a mapping request may be initiated by and decided upon other models and their ontologies, too (the dotted arrows from block 'Other Models' in Figure 3). For example, information about a user's daily agenda, such as Lovett et al.'s (2010) shared online calendar, or location-based activities (e.g., Dearman and Truong 2010, Dearman et al. 2011), may trigger the suspension of location updates if the user must not be disturbed. A privacy setting obtained from this user's privacy preferences may request to change the visualisation mode (Tang et al. 2011) or even remove location information within a certain area permanently (Brush et al. 2010).

The next section reports on the results of a real-life field study, in which we evaluated the effect of the described approach.

\section{Field study}

The two goals of our real-life field trial were 1) to investigate the influence of awareness of the uncertainty about users' localisation on their performance and behaviour in a map-assisted chasing task, and 2) to evaluate the individual importance of the corresponding visualisation patterns in providing this awareness.

\subsection{Environment, software and facilities}

The study was conducted on a Samsung Google Nexus S Android smartphone in a combined indoor-outdoor environment. The indoor segment included a part of the

This is a preprint of an article whose final and definitive form has been published in the Journal of Location Based Services (C) 2012 Taylor \& Francis; Journal of Location Based Services is available online at: http://www.tandfonline.com/doi/abs/10.1080/17489725.2012.682098 
university building where a WiFi-based localisation system was implemented and configured to locate users every 4 seconds. The outdoor segment covered the campus area and was tracked by GPS that reported location approximately every 4 seconds. Note that GPS signals were also sometimes available indoors through the glass roof or near the windows. Those situations, as well as the ones when the users either entered or left the indoor area intentionally, were processed by a handover mechanism. The handover partially used Hansen et al.'s (2009) results and considered the following aspects: a) a localisation system's reliability measure, i.e. the system had to produce at least two continuous readings in order to become a candidate for the handover; $b$ ) system's accuracy, i.e. if both systems were considered reliable in terms of aspect a), then the one with a better accuracy was set as active. This approach successfully handled both cases: occasional GPS signals indoors and when entering or leaving the building intentionally. The id of the tracking system that produced this smartphone user's last known (i.e. the most recently received) location was always displayed in the top left corner.

We used Google Maps (http://www.google.com/mobile/maps/) to show both outdoor and indoor locations (Figure 5 (left)). The options menu allowed the smartphone user to see the locations of the tracked users on the smartphone's screen or to zoom in to the user's own location. When zoomed in further beyond the Google Maps maximum zoom-level, an option that some users may need in the proximity of the indoor environment in order to explore further details of the building, the building layout in finer details was displayed. Namely, it showed a map of the entire building and of its zoomed-in segment, where the details of the users' locations were visualised in the appropriate colours (see Figure 5 (right)). When either tracked user joined or left

This is a preprint of an article whose final and definitive form has been published in the Journal of Location Based Services (C) 2012 Taylor \& Francis; Journal of Location Based Services is available online at: http://www.tandfonline.com/doi/abs/10.1080/17489725.2012.682098 
the displayed indoor map, a corresponding notification was shown on the screen and that user's visualisation generated or removed, respectively (Figure 6). While within the provided detailed building map, users could pan both maps at their convenience and zoom out if they wanted to return to the Google Maps mode (Figure 5 (left)). This limit of panning to within the building map was justified by the nature of the experiment that was designed for two users and covered the close proximity of the indoor environment; hence no context with respect to other users was lost. In section 6 we will discuss extensions on the visualisation for multi-user cases, where spatially distributed users must be visualised simultaneously within the context of the same screen. Extending the panning to outside of the detailed building map should in this case normally display the appropriate area of Google Maps at the corresponding zoom-level.

Using the smartphone with the above described application, the participants of the study had to meet a dedicated person in the case of no direct communication available between the two, i.e. using only the information shown on the smartphone's screen. The dedicated person (hereafter referred to as the target) was also equipped with a smartphone running the application. Note that we also took into account Seager and Stanton Fraser's (2007) finding, which revealed that the users of map-based navigation applications similar to ours preferred physical rotation of their mobile device to align the displayed map with their walking direction.

This is a preprint of an article whose final and definitive form has been published in the Journal of Location Based Services (C) 2012 Taylor \& Francis; Journal of Location Based Services is available online at: http://www.tandfonline.com/doi/abs/10.1080/17489725.2012.682098 
Figure 5. The application shows the locations of both the participant and the target in the corresponding colours on a map. When zoomed in further in the proximity of the indoor area, a map of the building layout in finer details is displayed. . The localisation system that produced the last known own location is displayed in the top left corner of the screen.
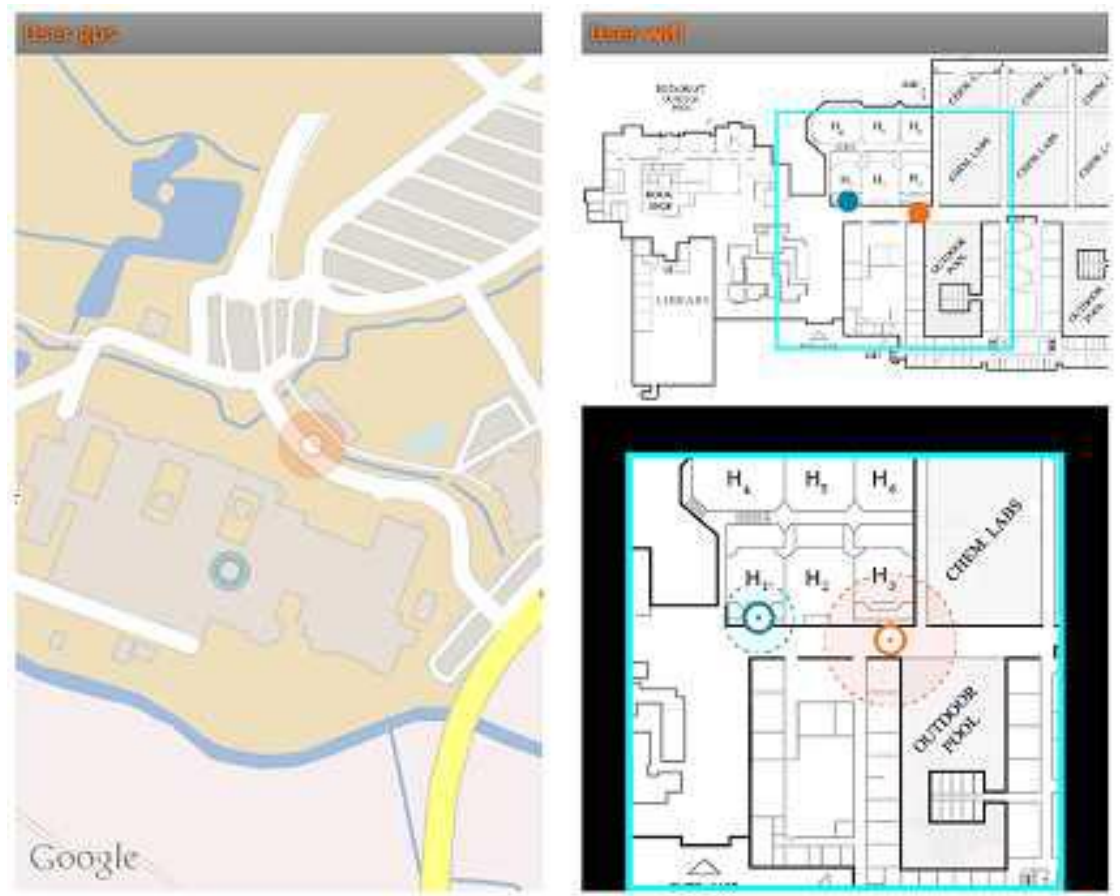

Figure 6. An appropriate notification is shown (and the visualisation is updated) if either the participant or the target joins or leaves the indoor environment.

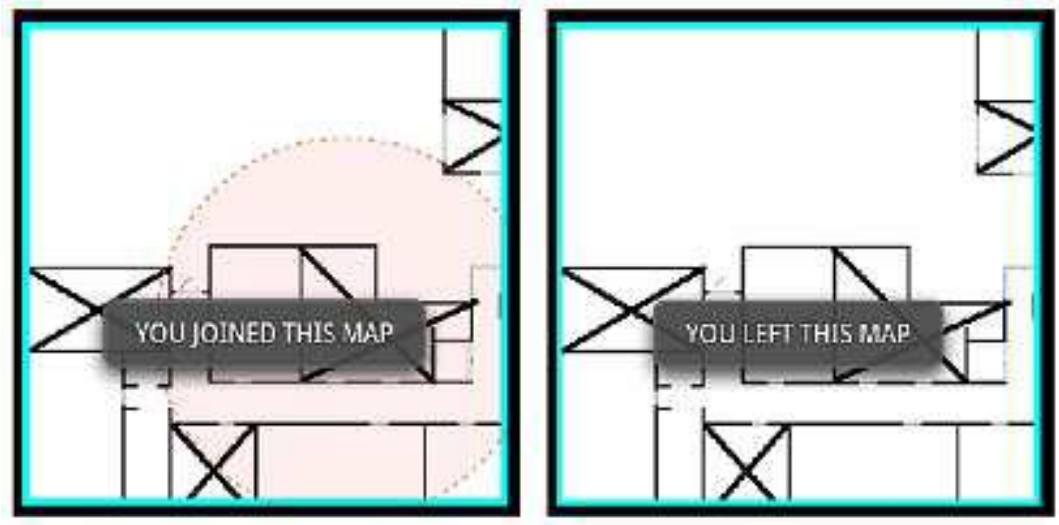

\subsection{Participants}

Ten people ( $7 \mathrm{~m}, 3 \mathrm{f})$, aged between 24 and $60(M=34$, median=30.5), participated in the

This is a preprint of an article whose final and definitive form has been published in the Journal of Location Based Services (C) 2012 Taylor \& Francis; Journal of Location Based Services is available online at:

http://www.tandfonline.com/doi/abs/10.1080/17489725.2012.682098 
study. They were all employees of our university, specialised in diverse fields, and were not involved in our research. We asked the participants to rank their expertise in 1) using map-based applications for navigation, and 2) working with smartphones. The provided scores on a 5-point Likert-type scale - 1-none, 2-a bit, 3-some, 4-quite a bit, 5a lot of - averaged to 2.9 (median=3) and 2.1 (median=1.5), respectively. The participants also ranked to what level they were familiar with the campus area and the building interiors $(M=3.7$, median=3.5).

\subsection{Procedure}

The study began with a briefing, during which we explained the participants the goal of the study, introduced the "chasing" task and provided the main instructions on the application usage and functionality. The participants also met the target.

We compared two approaches to visualising location. In one, a user's last received location and its errors are shown (Figure 3(a)). The other visualisation extends the first one by providing additional feedback on the tracking status of each location as it is described in section 3.1 and illustrated in Figure 3(a-d). The main part of the study thus comprised two sessions, one for each visualisation approach. We will hereafter refer to the sessions as the simple session and the extended session, respectively to the visualisation used in each session. In the beginning of each session, we additionally explained the behaviour of the corresponding visualisation. The participants then had to walk to an indicated starting area outdoors, from where they began the "chasing".

\subsubsection{Wizard-of-Ozfor manipulating the uncertainty}

Naturally, one's movements in a chasing task in a large-scale environment should be

This is a preprint of an article whose final and definitive form has been published in the Journal of Location Based Services (C) 2012 Taylor \& Francis; Journal of Location Based Services is available online at:

http://www.tandfonline.com/doi/abs/10.1080/17489725.2012.682098 
unrestricted as long as the corresponding areas are open to public. Therefore we let the participants plan their chasing strategy and navigation path individually in a way they felt most comfortable with.

Since location tracking was steadily available throughout the environment, we introduced artificial control over its availability so as to facilitate the tested conditions. Assigning a predefined static area may not work in the case of a freely chosen trajectory, for the latter might simply not run across that area. Therefore we simulated the absence of the participant's and the target's location updates using a wizard-of-oz (WOz) approach. The WOz, played by the experimenter, manually chose a suspension area at run-time using the information about the locations of both parties. A candidate area for suspension had to satisfy one criterion: the target had to be roughly in the centre of the area at the moment of suspension. Together with suspending the target's location updates, the WOz notified him about it with a vibrating alarm, and the target then proceeded to a previously agreed area (hereafter 'second area') located out of sight from the current one. The target's location updates were brought back on the participant's smart-phone as soon as the former left the suspension area and was on the way to the second area. It allowed the participant to see the target's locations again and thus to resume informed chasing. If the participant also entered the suspension area, the WOz suppressed their location updates as well while in the area.

We limited the areas where the suspension was possible to indoor locations in both sessions. This was done in order to avoid a direct line of sight between the participant and the target in an open area outdoors, i.e. when the participant could spot the target from a distance and thus ignore the application. Besides, Benford et al. (2006) found out previously that users of location-aware navigation applications tend to

This is a preprint of an article whose final and definitive form has been published in the Journal of Location Based Services (C) 2012 Taylor \& Francis; Journal of Location Based Services is available online at: http://www.tandfonline.com/doi/abs/10.1080/17489725.2012.682098 
remember such problematic areas during their later experience in the same environment and may, for example, exploit these areas in the future for tactical advances or try to shun them. To avoid such effect of possible learning about the first session's "bad" area, a different and previously unvisited area was suspended during the second session.

Each session completed when the participant met the target in the second area. On average, participants spent one hour to complete both sessions and answer all questions.

\subsubsection{Thinking aloud and supervision}

Chasing a moving object in a large-scale and crowded area entails different pathchoosing strategies. For example, the speed of walking, the experience of using mobile maps, the topology of the environment are among the many factors that may influence the chasing process. As we were mainly interested in analysing whether, how and in which situations visual awareness of uncertainty about localisation would be influential, we did not set any time constraints regarding the task completion to the participants. Instead, we instructed them to walk at a comfortable pace so that they would be able to pay enough attention to the information on the smartphone's screen. By doing so, we were also able to manage the "thinking aloud" (TA) approach during the sessions, in which the participants' oral comments were recorded and their behaviour observed for later analysis. We followed the speech-communication TA protocol (adopted from Olmsted-Hawala et al. 2010), in which the WOz acted as an active listener, replying a short and non-directive "um-hum" to the participant's TA comments. The same pattern, pronounced with a rising intonation, was used to remind the participant to keep TA after 15-20 seconds of silence. Walking next to the participant also allowed the WOz to

This is a preprint of an article whose final and definitive form has been published in the Journal of Location Based Services (C) 2012 Taylor \& Francis; Journal of Location Based Services is available online at:

http://www.tandfonline.com/doi/abs/10.1080/17489725.2012.682098 
observe the details of the participants' behaviour at run-time. Besides, due to the largescale and spatially distributed nature of the experiment, the WOz was able to take appropriate decisions on the session flow in case of technical problems or difficulties (e.g., terminating the session if the participant was stuck and wished to withdraw or the application behaved inappropriately), but no other communication or interference into the evaluation process was allowed. Only two participants had prior TA experience so an appropriate explanation of the above conditions was provided.

\subsection{Data collection}

We collected several types of data: run-time information, post-session questionnaires, a post-experiment evaluation block, and post-session and post-experiment comments in free form. The run-time information comprised the "think aloud" comments and indirect observations and comments the WOz noted about the behaviour and experience of the participants during the sessions. The post-session questionnaire evaluated the participants' experience after each session and included the following questions to be ranked on a 5-point Likert scale (the final question had a 3-point scale):

- How difficult was this session to complete? (1-not at all, 5-very difficult);

- How helpful in achieving your goal was the information about the users' locations? (1-not at all, 5-very helpful);

- How distracting from achieving your goal was the information about the users' locations? (1-not at all, 5-very distracting);

- How confusing was the information about the users' locations? (1-not at all, 5very confusing);

This is a preprint of an article whose final and definitive form has been published in the Journal of Location Based Services (C) 2012 Taylor \& Francis; Journal of Location Based Services is available online at: http://www.tandfonline.com/doi/abs/10.1080/17489725.2012.682098 
- How frustrating (annoying, stressing, discouraging, irritating) was the information about the users' locations? (1-not at all, 5-very frustrating (annoying, stressing, etc.));

- During the session, you referred to the information about the users' locations considerably (1-strongly disagree, 2-disagree, 3-neutral, 4-agree, 5-strongly agree);

- (if relative) How important was the fact that you were familiar with the environment? (1-not at all, 5-very important);

- (if relative) Would you use this type of users' run-time locations in a similar task in an unfamiliar environment? (Yes, No, Difficult to say).

The post-experiment evaluation block measured the usefulness of the visualisation patterns in the extended session in terms of how they assisted in the chasing task during this session. We asked the participants to rank how much they agreed with the following statements below, using a 5-point Likert scale (1-strongly disagree, 2-disagree, 3neutral, 4-agree, 5-strongly agree):

(1) The dot was helpful;

(2) The extending circle was helpful;

(3) The cross was helpful.

Since the performance in a large-scale environment may be influenced by a lot of factors indeed, we also asked the participants to support each score with a short explanation. This supplementary information was necessary in order to reveal whether the given score was influenced by external factors (i.e. unrelated to the awareness, such

This is a preprint of an article whose final and definitive form has been published in the Journal of Location Based Services (C) 2012 Taylor \& Francis; Journal of Location Based Services is available online at: http://www.tandfonline.com/doi/abs/10.1080/17489725.2012.682098 
as being dissatisfied with poor tracking, hurdles in the facilities, etc.) which, if not found out, could have led to a misinterpretation of the results.

Finally, the participants could also provide any additional comments and suggestions they had.

\section{Results and observations}

We derived the following main findings from the study:

- The extended design with the visualisation patterns, introduced in section 3.1 , is equally easy to use as the basic circle-based one (see Figure 1), and additional awareness of the uncertainty about location is beneficial;

- Different constituents of this awareness are of different importance; therefore the corresponding visualisation patterns should be given different priorities when designing a user interface that contains uncertainty about location;

- The importance depends on user profiles (such as their eyesight level, navigation skills, ability to understand maps, etc), distance between the users, and the quality and reliability of the tracking.

We will now describe and discuss the results and the above findings in detail.

\subsection{Overall performance}

Eight participants successfully completed both sessions. One participant (P8) could not complete the simple session due to an issue with the network that led to the application on the target's smartphone being unable to resume sending location updates after the target had left the suspension area. This, however, influenced P8's experience during

This is a preprint of an article whose final and definitive form has been published in the Journal of Location Based Services (C) 2012 Taylor \& Francis; Journal of Location Based Services is available online at: http://www.tandfonline.com/doi/abs/10.1080/17489725.2012.682098 
the extended session and brought in several valuable observations for later analysis. One participant (P7) could not complete both sessions: the target could not follow the instructions due to the rain that intensified after each session had already started, so he did not walk to the agreed second meeting area outdoors. The WOz was unaware of the target's incomplete path and therefore made a mistake in the suspend-resume process. This resulted in highly incorrect positioning so that the participant could not find the target and gave up. We had to discard P7's results because he rather evaluated the impact of erroneous information.

Figure 7(a-g) compares the scores provided to the per-session questions. The Wilcoxon signed-rank test applied to the scores didn't reveal any difference between the two designs, what suggests that the extended design comprising the additional three visualisation patterns is at least as good to use as the basic one (see Figure 1). This allows us to consider and analyse the added value the visualisation patterns bring into the extended visualisation.

This is a preprint of an article whose final and definitive form has been published in the Journal of Location Based Services (C) 2012 Taylor \& Francis; Journal of Location Based Services is available online at:

http://www.tandfonline.com/doi/abs/10.1080/17489725.2012.682098 
Figure 7. A: Comparison of the 1-5 Likert scale scores to the questions after each session (left - simple session, right - extended session): (a) - session difficulty, (b) helpfulness of location information, (c) - distraction level, (d) - confusion level, (e) frustration level, (f) - reference to location information, $(\mathrm{g})$ - familiarity with the test environment; B: The 1-5 Likert scale scores for the involved visualisation patterns in the extended session: (1) - dot helpful, (2) - extending circle helpful, (3) - cross helpful.

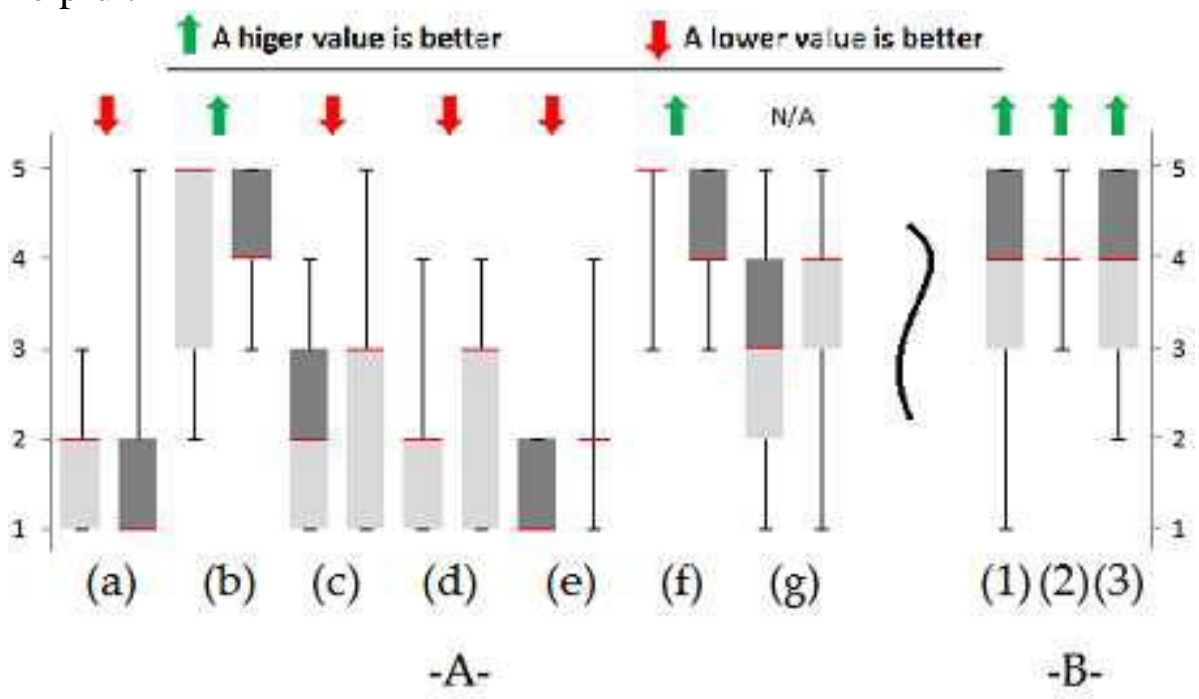

All participants referred to the additional awareness in all occasions where they recognised and experienced it during the extended session. The results in Figure 7(1-3) show that regardless of the experience during the tasks, all three involved visualisation patterns were considered helpful with respect to the information they were intended to provide:

- Dot $($ mean=3.7, median=4, $\boldsymbol{\sigma}=\mathbf{1 . 4 1})$. Three participants, when explaining the corresponding score, commented that they could not see the dot or just did not pay attention to it so they simply did not notice it at all (hence a higher $\sigma$ value). The rest (strongly) agreed that it was useful. "I like the heart bit of my location now." - P6, "Status indication of signal assured correctness of location." - P8. In particular, because of the negative experience during the simple session, $\mathrm{P} 8$

This is a preprint of an article whose final and definitive form has been published in the Journal of Location Based Services (C) 2012 Taylor \& Francis; Journal of Location Based Services is available online at: http://www.tandfonline.com/doi/abs/10.1080/17489725.2012.682098 
indicated that he was especially concerned that the dot be on, "[I was] afraid of missing signal indication reliability."

- Circle $(\operatorname{mean}=4$, median=4, $\boldsymbol{\sigma}=\mathbf{0 . 7})$. Two participants expressed that they did not refer to the extending nature of the circle much and therefore could not conclude that that property assisted them. However, they did explore the area of the map covered by the circle. For example, P5 realised that the target, while being shown indoors, was actually outdoors, i.e. near the circle's edge (see Figure 8). The rest regarded the dynamically extending circle as a useful indication of the location tracking dynamics. "The first [extended] session was easier to use because of some continuous changes." $-P 2$.

Figure 8. One of the participants realised that the target, while being shown indoors, was actually outdoors, i.e. near the circle's edge, so she left the auditorium and proceeded towards the nearest exit, following the map.

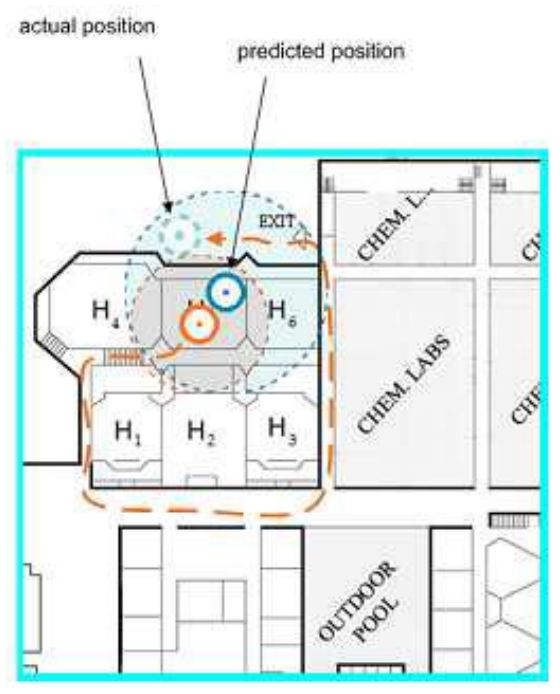

- Cross $($ mean=3.9, median=4, $\sigma=1.05)$. Overall, the cross served the intended means of awareness - to communicate the information about an outdated (missing) location - as in "I can see target not there, so change strategy to

This is a preprint of an article whose final and definitive form has been published in the Journal of Location Based Services (C) 2012 Taylor \& Francis; Journal of Location Based Services is available online at: http://www.tandfonline.com/doi/abs/10.1080/17489725.2012.682098 
locate target" - P4, or "Unavailability of signal for a longer time indicates that we cannot rely on the location anymore" - P8. P5's negative score (2/5) was brought by her confusion about the time when she stopped receiving updates from the target, "It confused me that he [the target] disappeared, so how can it [the cross] be helpful?!" - P5. We clarify on this response further on below when we talk about the cross' importance.

\subsection{Individual evaluation}

Each visualisation pattern turned out to be of different importance. The dot indicating that the location update was recent was ignored by the participants who could not focus or felt uncomfortable focusing on that detail. Also, it was mainly invisible outdoors in the sunlight. Although the participants did not reflect this fact in the ranking, they did mention it in the beginning of a sunny session. Moreover, since the participants started outdoors and the target remained indoors in the beginning of the session, then, prior to entering the building, they often carried the device in their lowered arm and only occasionally raised the hand up to look at the screen. Thus the importance of the dot (given that it can be perceived if shown) increases in problematic areas where location tracking may become unreliable or absent, or in situations when participants are close to each other and consult the map more often.

In a similar way, participants paid less attention to the circle's gradual extension while location tracking was stable. However, its importance also increased in the problematic areas, what can be illustrated by an observation linked to the target's suspended period in the simple session. In that case, while no updates were made to the visualisation of the target's position, his resumed updates outside of the suspended area

This is a preprint of an article whose final and definitive form has been published in the Journal of Location Based Services (C) 2012 Taylor \& Francis; Journal of Location Based Services is available online at:

http://www.tandfonline.com/doi/abs/10.1080/17489725.2012.682098 
surprised the participants, for the new position appeared at quite a distance from the last known (i.e. prior to the suspension) location - "What was it? Did he move so fast or what?" - P3, "He moved!" (a remark during the session), "It was a bit annoying that in one second the object was in one position and just after it [he] moved without visible movement" (a comment to the ranking) - P9, "Predicted spot was flipping - sudden location change" - P10. And the extending circle gradually transforming and replaced, after a time, with a cross gave the feeling of movement and dynamics and created a firm positive attitude. In other words, it prepared the participants for the change, smoothing the cause of no location tracking.

In the case of lost tracking, i.e. when the circle eventually turned into a cross, the participants reacted differently. For example, P4 tried to estimate where he should look now and did not go to where the target's cross was shown. P6 admitted that she was just guessing. P9 did nothing, stood still and waited for the target's location to resume. Somewhat apart here stays P5's reaction, who disagreed that the cross was helpful. But when we asked her to explain the given score, it became apparent that it was the very fact that the target could not be tracked that made her unhappy (see the quote above), but the awareness about it was recognised and accepted (which she did notice as can be seen from her comment about that moment, "How come is he here? Hmm...Oops, the cross now. Did he disappear?!'). Besides, she agreed that seeing the blinking dot indicating that the location tracking was active was also very helpful (5-strongly agree). Therefore, we conclude that the cross for the case of missing tracking updates was important for the awareness and should always be activated.

This is a preprint of an article whose final and definitive form has been published in the Journal of Location Based Services (C) 2012 Taylor \& Francis; Journal of Location Based Services is available online at: http://www.tandfonline.com/doi/abs/10.1080/17489725.2012.682098 


\subsection{Other findings}

None of the participants refused to try the task in a completely unfamiliar environment, with 7 out of 9 agreeing to use the extended design, of which 5 would also try the simple one. Moreover, P10 supported his "Difficult to say" choice by saying that he never needed that kind of information in his activities, but nevertheless he expressed an interest in repeating the test in an environment unknown to both parties. Similarly, P4 concluded that knowing the environment was not very important to him with respect to completing the task ("I think environment is not important factor; using this [extended] scenario, target's changing location can be seen on map, and more info than before [simple]"); therefore he was also curious to validate his scores in an unfamiliar environment.

There were situations, in which additional awareness, though recognised, was rather bewildering. For example, P8 in his failed simple session got perplexed by not having found the target even within the precision area. P2 admitted that seeing the additional awareness was quite helpful as it allowed knowing more about the movements. But at the same time he explicitly mentioned that the perplexity in the extended session was caused by imprecise GPS positioning near the entrance (he stood quite close to the wall), so he preferred the simple mode because of more accurate location detection. Such observations of reacting to the application's inappropriate behaviour, in fact, are related to the problem of intelligibility in context-aware applications (e.g., "Why is my GPS position so far from where I actually am?" - "You are standing too close to the building"), where Lim and Dey (2011) showed recently that providing intelligibility increased users' impressions about a context-aware application with low certainty when it behaved inappropriately.

This is a preprint of an article whose final and definitive form has been published in the Journal of Location Based Services (C) 2012 Taylor \& Francis; Journal of Location Based Services is available online at: http://www.tandfonline.com/doi/abs/10.1080/17489725.2012.682098 


\section{Conclusions}

We presented an approach to incorporate awareness and uncertainty of location for differing magnitudes of covered surfaces in mobile graphical user interfaces. The user interface adaptations to present diversified levels of awareness and uncertainty are accomplished by mapping the capabilities of localisation systems and location data on an ontology that can be used for reasoning. The ontology is used to produce a set of user interface elements, called visualisation patterns, which represent the characteristics of uncertainty and can be used as part of a complete user interface.

We evaluated our approach in a field study, in which participants, assisted by a smartphone running a map-based application, completed two tasks under varying location tracking conditions in the suggested indoor-outdoor environment. In each task, the uncertainty was visualised according to either the referred basic technique, which previously proved to be beneficial for navigation tasks (Dearman et al. 2007), or the experimental design under investigation. The results of the study show that the extended design turns out to be at least as good to use as the basic one, i.e. the additional visual information is not considered a burden. The evaluation of each visualisation pattern reveals their impact depends on each user's personal profile (such as their eyesight level, navigation skills, ability to understand maps, etc.), distance between the users (knowing about finer details of someone else's state is less important if they are far), and the quality and reliability of the tracking. Based on these conclusions and observations, we identify a set of guidelines for presenting the uncertainty of location in a user interface.

Our visualisation patterns and strategies are candidates for usage in multi-user applications. The amount of location information users are interested in or willing to This is a preprint of an article whose final and definitive form has been published in the Journal of Location Based Services (C) 2012 Taylor \& Francis; Journal of Location Based Services is available online at: http://www.tandfonline.com/doi/abs/10.1080/17489725.2012.682098 
give is greatly affected by various details, often considered in combination with each other (Reilly et al. 2006). This will require visualisations, where the degree of correctness depends on the user's willingness to share the corresponding details. Therefore the future work will focus on incorporating users' social profiles, their personal preferences or agendas (see section 3.3 for the discussion and examples) into the adaptation model.

\section{Acknowledgements}

Part of the research at EDM is funded by EFRO (European Fund for Regional Development) and the Flemish Government. Funding for this research was also provided by the Research Foundation - Flanders (F.W.O. Vlaanderen, project CoLaSUE, number G.0439.08N).

\section{References}

Aksenov, P., Luyten, K., and Coninx, K., 2011. A unified scalable model of user localisation with uncertainty awareness for large-scale pervasive environments. In Proceedings of the 5th International Conference on Next Generation Mobile Applications, Services and Technologies, pages 212-217.

Anastassova, M., Magnusson, C., Pielot, M., Randall, M., and Claassen, G.B., 2010. Using audio and haptics for delivering spatial information via mobile devices. In Proceedings of the 12th international conference on Human Computer Interaction with Mobile Devices and Services, pages 525-526.

Banerjee, N., Agarwal, S., Bahl, P., Chandra, R., Wolman, A., and Corner, M., 2010. Virtual Compass: relative positioning to sense mobile social interactions. In Proceedings of the 8th International Conference on Pervasive Computing, pages $1-21$.

This is a preprint of an article whose final and definitive form has been published in the Journal of Location Based Services (C) 2012 Taylor \& Francis; Journal of Location Based Services is available online at:

http://www.tandfonline.com/doi/abs/10.1080/17489725.2012.682098 
Baus, J. and Kray, C., 2002. Frames of reference, positional information and navigational assistance. In Proceedings of the 15th International Florida Artificial Intelligence Research Society Conference, pages 461-465.

Benford, S. et al., 2006. Can you see me now?. ACM Transactions on HumanComputer Interaction, 13(1), 100-133.

Brush, A.J., Krumm, J., and Scott, J., 2010. Exploring end user preferences for location obfuscation, location-based services, and the value of location. In Proceedings of the 12th International Conference on Ubiquitous Computing, pages 95-104.

Burigat, S. and Chittaro, L., 2011. Pedestrian navigation with degraded GPS signal: investigating the effects of visualizing position uncertainty. In Proceedings of the 13th International Conference on Human Computer Interaction with Mobile Devices and Services, pages 221-230.

Cearreta, I. and Garay-Vitoria, N., 2011. Toward adapting interactions by considering user emotions and capabilities. In Proceedings of the 14th International Conference on Human-Computer Interaction, pages 525-534.

Damián-Reyes, P., Favela, J., and Contreras-Castillo, J. 2011. Uncertainty management in context-aware applications: increasing usability and user trust. Wireless Personal Communications, 56(1), 37-53.

Dearman, D., Hawkey, K., and Inkpen, K., 2005. Rendezvousing with location-aware devices: enhancing social coordination. Interacting with Computers, 17(5), 542566.

Dearman, D., Sohn, T., and Truong, K.N., 2011. Opportunities exist: continuous discovery of places to perform activities. In Proceedings of the 29th Conference on Human Factors in Computing Systems, pages 2429-2438.

Dearman, D. and Truong K.N., 2010. Identifying the activities supported by locations with community-authored content. In Proceedings of the 12th International Conference on Ubiquitous Computing, pages 23-32.

Dearman, D., Varshavsky, A., de Lara, E., and Truong, K.N., 2007. An exploration of location error estimation. In Proceedings of the 9th International Conference on Ubiquitous Computing, pages 181-198.

This is a preprint of an article whose final and definitive form has been published in the Journal of Location Based Services (C) 2012 Taylor \& Francis; Journal of Location Based Services is available online at:

http://www.tandfonline.com/doi/abs/10.1080/17489725.2012.682098 
Gellersen, H., Lukowicz, P., Beigl, M., and Riedel, T., 2010. Cooperative relative positioning. IEEE Pervasive Computing, 9(4), 78-89.

Hansen, R., Wind, R., Jensen, C.S., and Thomsen, B., 2009. Seamless indoor/outdoor positioning handover for location-based services in Streamspin. In Proceedings of the 10th International Conference on Mobile Data Management, pages 267272.

Hervás, R. and Bravo, J., 2011. Towards the ubiquitous visualization: Adaptive userinterfaces based on the Semantic Web. Interacting with Computers 23(1), 4056.

Kjærgaard, M.B., Blunck, H., Godsk, T., Toftkjær, T., Christensen, D.L., and Grønbæk, K., 2010. Indoor positioning using GPS revisited. In Proceedings of the 8th International Conference on Pervasive Computing, pages 38-56.

Langdal, J., Kjærgaard, M.B., Toftkjær, T., and Schougaard, K., 2010. Exposing position uncertainty in middleware. In Proceedings of the 2nd International Workshop on Middleware for Pervasive Mobile and Embedded Computing, pages $1-8$.

Lemelson, H., King, T., and Effelsberg, W., 2008. A study on user acceptance of error visualization techniques. In Proceedings of the International Workshop on Human Control of Ubiquitous Systems.

Lim, B., and Dey, A., 2011. Investigating intelligibility for uncertain context-aware applications. In Proceedings of the 13th International Conference on Ubiquitous Computing, pages 415-424.

Lovett, T., O’Neill, E., Irwin, J., and Pollington, D., 2010. The calendar as a sensor: analysis and improvement using data fusion with social networks and location. In Proceedings of the 12th International Conference on Ubiquitous Computing, pages 3-12.

Marquardt, N., Gross, T., Carpendale, M.S.T., and Greenberg, S., 2010. Revealing the invisible: visualizing the location and event flow of distributed physical devices. In Proceedings of the Fourth International Conference on Tangible, Embedded, and Embodied Interaction, pages 41-48.

This is a preprint of an article whose final and definitive form has been published in the Journal of Location Based Services (C) 2012 Taylor \& Francis; Journal of Location Based Services is available online at:

http://www.tandfonline.com/doi/abs/10.1080/17489725.2012.682098 
Misund, G., Holone, H., Tolsby, H., Karlsen, J., and Toppe, A., 2009. Effects of coplayer visualization in a location-based chase-and-catch game. In Proceedings of the 11th International Conference on Human Computer Interaction with Mobile Devices and Services.

Nova, N., Girardin, F., and Dillenbourg, P., 2010. The effects of mutual locationawareness on group coordination. International Journal of Human-Computer Studies, 68(7), 451-467.

Olmsted-Hawala, E.L., Murphy, E., Hawala, S., and Ashenfelter, K.T., 2010. Thinkaloud protocols: a comparison of three think-aloud protocols for use in testing data-dissemination web sites for usability. In Proceedings of the 28th Conference on Human Factors in Computing Systems, pages 2381-2390.

Ongenae, M., et al., 2008. Design of a semantic person-oriented nurse call management system. International Journal of Web and Grid Services, 4(3), 267-283.

Patricia, 2010. Average human walking speed: walking for racing | race walking | speed of walking [online]. Yoga Health Benefits. Available from: http://www.yogawiz.com/blog/walking/average-human-walking-speed.html [Accessed 05 December 2011].

Pulkkinen, T., Bhattacharya, S., and Nurmi, P., 2011. Abstracting positions for indoor location based services. In Proceedings of the IUI Workshop on Location Awareness for Mixed and Dual Reality.

Ranganathan, A., Al-Muhtadi, J., Chetan, S., Campbell, R., and Mickunas, M.D., 2004. MiddleWhere: a middleware for location-awareness in ubiquitous computing applications. In Proceedings of the 5th International Conference on Middleware, pages $397-416$.

Reilly, D., Dearman, D., Ha, V., Smith, I., and Inkpen, K., 2006. "Need to know": examining information need in location discourse. In Proceedings of the 4th International Conference on Pervasive Computing, pages 33-49.

Seager, W. and Stanton Fraser, D., 2007. Comparing physical, automatic and manual map rotation for pedestrian navigation. In Proceedings of the 25th Conference on Human Factors in Computing Systems pages 767-776.

This is a preprint of an article whose final and definitive form has been published in the Journal of Location Based Services (C) 2012 Taylor \& Francis; Journal of Location Based Services is available online at: http://www.tandfonline.com/doi/abs/10.1080/17489725.2012.682098 
Stevenson, G., Ye, J., Dobson, S., and Nixon, P., 2010. LOC8: A location model and extensible framework for programming with location. IEEE Pervasive Computing, 9(1), 28-37.

Strobbe, M. et al., 2007. Design of CASP: an open enabling platform for context aware office and city services. In Proceedings of the Fourth International Workshop on Managing Ubiquitous Communications and Services, pages 123-142.

Tang, K., Hong, J., and Siewiorek, D.P., 2011. Understanding how visual representations of location feeds affect end-user privacy concerns. In Proceedings of the 13th International Conference on Ubiquitous Computing, pages 207-216.

Varshavsky, A. and Patel, S., 2009. Location in ubiquitous computing. In J. Krumm, ed. Ubiquitous computing fundamentals. Boca Raton, FL: Chapman and Hall/CRC, $285-320$

Wagner, J. and Kray, C., 2010. Opportunistic peer-to-peer pedestrian positioning system. In Poster at the 8th International Conference on Pervasive Computing.

This is a preprint of an article whose final and definitive form has been published in the Journal of Location Based Services (C) 2012 Taylor \& Francis; Journal of Location Based Services is available online at:

http://www.tandfonline.com/doi/abs/10.1080/17489725.2012.682098 